Research Paper

\title{
Expression of Chosen Carcinoembryonic-Related Cell Adhesion Molecules in Pancreatic Intraepithelial Neoplasia (PanIN) Associated with Chronic Pancreatitis and Pancreatic Ductal Adenocarcinoma (PDAC)
}

Justyna Zińczuk ${ }^{\circledR}$, Konrad Zaręba², Wioletta Romaniuk³ ${ }^{3}$, Dorota Kamińska4 ${ }^{4}$, Marcin Nizioł5 , Magdalena Baszun ${ }^{5}$, Bogusław Kędra², Katarzyna Guzińska-Ustymowicz ${ }^{5}$, Anna Pryczynicz ${ }^{5}$

1. Department of Clinical Laboratory Diagnostics, Medical University of Bialystok, Waszyngtona 15 St., 15-269 Białystok, Poland

2. 2nd Clinical Department of General and Gastroenterological Surgery, Medical University of Bialystok, M. Skłodowskiej-Curie 24A St., 15-276 Białystok, Poland

3. Department of Haematology, Medical University of Bialystok, M. Skłodowskiej-Curie 24A St., 15-276 Białystok, Poland

4. Department of Laboratory Diagnostics, Independent Public Health Care Unit of the Provincial Hospital Jędrzej Śniadecki in Bialystok, Poland

5. Department of General Pathomorphology, Medical University of Bialystok, Waszyngtona 13 St., 15-269 Białystok, Poland

$\square$ Corresponding author: Justyna Zińczuk, Department of Clinical Laboratory Diagnostics, Medical University of Bialystok, Email: j.zinczuk@wp.pl, Tel (fax): $+48(85) 8318716$

(c) Ivyspring International Publisher. This is an open access article distributed under the terms of the Creative Commons Attribution (CC BY-NC) license (https://creativecommons.org/licenses/by-nc/4.0/). See http://ivyspring.com/terms for full terms and conditions.

Received: 2019.01.02; Accepted: 2019.03.23; Published: 2019.04.25

\begin{abstract}
Aims: Carcinoembryonic antigen-related cell adhesion molecules (CEACAMs) are members of the glycosylphosphatidylinositol (GPI)-linked immunoglobulin (Ig) superfamily and take part in regulation of cell adhesion, tumor suppression and angiogenesis. Overexpression of CEACAM 1, 5 and 6 is widely described in several gastrointestinal epithelial tumors. The aim of study was to evaluate the expression of CEACAM 1, CEACAM 5 and CEACAM 6 in the most common precursor lesions of pancreatic ductal adenocarcinoma -pancreatic intraepithelial neoplasia (PanIN).

Methods and results: The study group consisted of 32 patients treated for chronic pancreatitis and 38 patients with pancreatic ductal adenocarcinoma who also had pancreatic intraepithelial neoplasia. The expression of CEACAM was performed by immunohistochemical method and evaluated using 3-point scale: 0 - lack of positive reaction in pancreatic intraepithelial neoplasia, 1 (weak and moderate) - reaction present in 1-30\% epithelial cells in PanIN and 2 (strong) - reaction present in $>30 \%$ epithelial cells in PanIN. Expression of CEACAM 1, 5 and 6 increased with increasing degree of advancement of PanIN. Differences in expression of CEACAM 1, 5 and 6 between normal pancreatic ducts and different degrees of PanIN were statistically significant $(p<0.001)$. We observed relationship between CEACAMI expression and localization of PanIN in different parts of the pancreas.

Conclusions: CEACAM 1, CEACAM 5 and CEACAM 6 expression appears to be an early event in pancreatic carcinogenesis. Moreover, expression of CEACAM 1, 5 and 6 may represent a useful biomarker that may aid in the identification of precancerous lesions in the pancreas.
\end{abstract}

Key words: pancreatic intraepithelial neoplasia, PanIN, CEACAM, adhesion molecules

\section{Introduction}

Pancreatic cancer is currently the third leading cause of cancer-related death in the United States and a major cause of morbidity and mortality worldwide. According to the American Cancer Society, the morbidity of pancreatic cancer will equal around 55440 and the mortality, about 44330 in 2018 year in the USA [1]. Such a high mortality is due to the late diagnosis and cancer resistance to chemotherapy and 
radiation [2]. Therefore, it is very important to detect cancer early, before it changes into an invasive form, when the possibility of complete cure is minimal.

The most common histological type of pancreatic cancer (over 95\% of cases) is pancreatic ductal adenocarcinoma (PDAC) which develops from pancreatic ductal epithelial cells[3]. Studies suggest that PDAC develops from precursor lesions pancreatic intraepithelial neoplasias (PanINs), intraductal papillary mucinous neoplasms (IPMNs) and mucinous cystic neoplasms (MCNs). The most frequently occurring and the best defined in the literature precursor lesion is pancreatic intraepithelial neoplasia defined as microscopic flat or papillary and noninvasive epithelial lesion developing in small pancreatic ducts $(<5 \mathrm{~mm}$ diameter). These lesions are composed of cuboidal or columnar cells with varying amounts of mucin facilitating their distinction from normal ductal epithelium composed of cuboidal or low columnar cells with amphophilic cytoplasm and without any evidence of mucinous cytoplasm [4]. According to the degree of architectural and cytological atypia in pancreatic ducts, PanINs are categorized into four grades: the lowest grade - PanIN 1A and PanIN 1B, PanIN 2 - intermediate grade PanINs and high grade PanIN - PanIN 3. PanIN 3 is the highest grade, also referred to as carcinoma in situ [5].

Carcinoembryonic antigen-related cell adhesion molecules (CEACAMs) belong to the glycosylphosphatidylinositol(GPI)-linked immunoglobulin (Ig) superfamily and are present on the apical surface of numerous cell types such as endothelial and epithelial cells of different organs [6]. According to the cell type and CEACAM subtype they may have different functions. Carcinoembryonic antigen-related cell adhesion molecules participate in the regulation of cell adhesion and cell cycle, in intracellular and intercellular signaling, cancer progression, inflammation, angiogenesis, and metastasis [7]. Recent studies revealed that some of CEACAM molecules, especially CEACAM 1,5 and 6 are valid clinical biomarkers and promising therapeutic targets in melanoma, colorectal, pancreatic and lung cancers [8-11].

CEACAM 1 (CD66a) has the widest tissue distribution of all members of CEACAM family. It is present on membrane either at the apical or lateral poles on different epithelial cells, endothelial cells, as well as in monocytes, granulocytes, activated T and B cells and a subset of natural killer cells [12]. Localization of this protein on cell membranes allows the connection to other surface receptors, extracellular matrix proteins, integrins and cytoskeletal elements [7]. Moreover, CEACAM 1 through the ability to respond to the activation of receptor Tyr kinases
(RTKs) may take part in the regulation of downstream signaling pathways and consequently may indirectly influence on cancer progression by stimulation of cellular proliferation, migration, invasion and metastasis, apoptosis, inflammation, immune evasion, angiogenesis induction, and genome instability $[7$, 13].

CEACAM 5 (CD66e) and CEACAM 6 (CD66c) similarly to CEACAM 1 are involved in homotypic interactions with other molecules belonging to CEA family and in heterotypic binding with integrin receptor $[14,15]$. Overexpression of CEACAM 5 and 6 inhibits anoikis which is an apoptotic response in normal cells to inadequate or inappropriate intercellular or matrix attachment [16]. In normal tissue, anoikis prevents ectopic cellular proliferation whereas tumor cells are characterized by resistance to anoikis [17]. It suggests that these intercellular adhesion molecules are involved in primary tumorigenesis and metastasis in cancers. Moreover, CEACAM 6 suppression by specific siRNA causes increased susceptibility to caspase-mediated anoikis, decreasing of Akt phosphorylation, and inhibits metastatic abilities [18]. CEACAM 5 is widely expressed in different types of normal tissues in the apical membranes of gland cells of the duodenum, parotid and submaxillary gland, colon, and stomach and is a tumor marker for several gastrointestinal and respiratory cancers [19]. Overexpression of CEACAM 6 has been observed in many human cancers such as colorectal, breast, pancreatic, mucinous ovarian, gastric, and lung cancers [20]. It was also present in precancerous lesions of colorectal cancer (colonic hyperplastic polyps and early adenomas) and was considered being the earliest molecular change observed in these lesions.

To date, role of CEACAM 1, 5 and 6 in development of precancerous lesions in the pancreas has not been explained. Therefore the aim of this study was to assess the expression of carcinoembryonic antigen-related cell adhesion molecules 1, 5 and 6, and to investigate their potential role in the development of pancreatic intraepithelial neoplasia.

\section{Material and methods}

\section{Patients}

The study group consisted of 32 patients treated for chronic pancreatitis and 38 patients with pancreatic ductal adenocarcinoma operated in the 2 nd Clinical Department of General and Gastroenterological Surgery at the University Hospital in Bialystok, in the years 2006-2014. The characteristics of the study group are shown in Table 1 . The study was performed in conformity with the Declaration of 
Helsinki for Human Experimentation and received approval by the Local Bioethics Committee of the Medical University of Bialystok (nr R-I-002/399/ 2016).

Table 1. Characteristics of the study group.

\begin{tabular}{ll}
\hline Clinicopathological features & Frequency, $\mathbf{n}(\%)$ \\
\hline Sex & \\
Male & $35(50 \%)$ \\
Female & $35(50 \%)$ \\
Age & \\
$<60$ years & $33(47.2 \%)$ \\
$\geq 60$ years & $37(52.8 \%)$ \\
Diagnosis & \\
pancreatitis & $32(32.9 \%)$ \\
pancreatic ductal adenocarcinoma & $38(54.3 \%)$ \\
Location & \\
head & $33(47.1 \%)$ \\
body & $5(7.1 \%)$ \\
tail & $20(28.6 \%)$ \\
body and tail & $12(17.2 \%)$ \\
\hline
\end{tabular}

\section{Histopathological examination and identification of ductal lesions}

The postoperative material was fixed in buffered formalin and paraffin-embedded. From paraffin blocks, 5- $\mu \mathrm{m}$ sections were cut and stained with hematoxylin-eosin $(\mathrm{H}+\mathrm{E})$. Histopathological analysis included diagnosis of primary disease, but also the presence and stage of pancreatic intraepithelial neoplasia which has been described in each patient. All slides were reviewed by two independent pathologists. PanIN lesions were classified according to the guidelines established by international group of pathologists on Pancreas Cancer Think Tank meeting sponsored by the National Cancer Institute and held in Park City, Utah in September 16-19, 1999 [21]. Briefly, PanIN 1A is an epithelial flat lesion whereas PanIN 1B is a papillary or micropapillary lesion composed of tall columnar cells with basally located nuclei and abundant supranuclear mucin without cytologic atypia. PanIN 2 is a mucinous, epithelial flat or papillary lesion with some nuclear abnormalities including loss of polarity, crowding, enlargement, nuclear stratification and hyperchromatism. PanIN 3 usually is papillary or micropapillary architecture with abnormal cribriforming, budding and luminal necrosis with cytologic abnormalities, such as loss of nuclear polarity, dystrophic goblet cells, atypical mitotic figures and macronucleoli [21]. The presence of PanINs was evaluated on the slides of the normal pancreatic tissue at least $5 \mathrm{~mm}$ away from the carcinoma, while, in the non-neoplastic lesions, PanINs were evaluated in the site of an ongoing disease process.

\section{Immunohistochemistry}

Tissue blocks were cut using a microtome into $4-\mu m$-thick sections on silanized glasses. The sections were deparaffinized in xylenes and hydrated in alcohols. In order to exhibit an antigen, the tissue sections were heated in a water bath at $99^{\circ} \mathrm{C}$ for 20 min and next cooled for $20 \mathrm{~min}$ in room temperature in citrate buffer $(\mathrm{pH}=6.0)$. Then, they were incubated with 3\% hydrogen peroxide to block endogenous peroxidase and, next, with protein block (Novocastra) for $5 \mathrm{~min}$. Incubation with CEACAM 1 mouse anti-human polyclonal antibody (clone VU-3C6, R\&D Systems) at a dilution of 1:100, CEACAM 5 mouse anti-human polyclonal antibody (clone 5B12, Sigma Aldrich) at a dilution of 1:100 and CEACAM 6 mouse anti-human polyclonal antibody (R\&D Systems) at a dilution of 1:100 for 60 minutes in room temperature. Following streptavidin-biotin reaction (biotinylated secondary antibody, streptavidin-HRP; Novocastra), the antigen antibody complex was visualized by application of chromogen 3,3'-diaminobenzidine (DAB, Novocastra). All slides were stained simultaneously with appropriate specimens, which served as positive controls. We used as a positive control cervical cancerous tissue for CEACAM1, colon cancerous tissue for CEACAM5 and colon and breast cancerous tissue were used as positive control for CEACAM6. Negative controls were performed by incubating the sample without the primary antibodies.

\section{Analysis of Immunohistochemical data}

Immunohistochemical assessment of CEACAM 1, 5 and 6 was performed in all pancreatic ducts in specimen from each patient. In the case of more than one type of PanIN lesions present in many ducts in each patient the authors described a mean value of expression of protein. Reaction was independently assessed by two senior pathologists who have been blinded to the clinicopathological data. In case of disagreement, the scoring was discussed by the pathologists until they agreed on a final result. Positive expression of CEACAM 1 and 5 was assessed in membrane of pancreatic ductal epithelial cells along the luminal cell border. In case of CEACAM 6 positive reaction also was assessed along the luminal cell border and in the cytoplasm of pancreatic ductal epithelial cells. The results of the staining were semiquantitatively assessed for the percentage of the positively stained cells. Expression of CEACAM 1, 5 and 6 was evaluated based on 3-point scale: 0 - lack of expression or weak positive reaction in less than $5 \%$ of cells, 1 (weak and moderate) - focal, positive reaction present in $5-25 \%$ of pancreatic ductal epithelial cells and 2 (strong) - diffuse, positive reaction present in $>25 \%$ of pancreatic ductal epithelial cells. 


\section{Statistical analysis}

Statistical analyses were performed using STATISTICA 10.0 (Statsoft, Cracow, Poland) and GraphPad Prism 7. The correlations of CEACAM proteins expression with clinicopathological parameters, and the significance of differences in CEACAM expression were determined by Pearson's chi-square test. All statistics were two-tailed with $\mathrm{p}$ value $\leq 0.05$ considered significant. Missing data were removed in pairs.

\section{Results}

\section{The evaluation of pancreatic intraepithelial neoplasia in patients with pancreatic ductal adenocarcinoma and pancreatitis}

In patients with pancreatic ductal adenocarcinoma the most common lesions was PanIN 1 (55.4\%) like as in group with pancreatitis (55.0\%). PanIN 2 in group with PDAC constituted $22.3 \%$ and PanIN 3 $11.5 \%$ of all founded lesions. Similar results were obtained in patients with pancreatitis in case of PanIN 2 which constituted 20.2\%. PanIN 3 occurred less frequently in patients with pancreatitis than in group with PDAC and constituted $5.5 \%$ of all lesions (Table 2).

\section{Analysis of CEACAM 1, 5 and 6 expression in relations to clinicopathological parameters}

The positive immunohistochemical reaction of CEACAM 1 and 5 was observed in membrane of pancreatic ductal epithelial cells along the luminal cell border. In case of CEACAM 6 positive reaction also was present along the luminal cell border and in the cytoplasm of pancreatic ductal epithelial cells (Figure 1,2 , 3). In normal pancreatic ducts staining for CEACAM 1, 5 and 6 was negative.

Table 2. PanIN assessed in the group of patients with pancreatitis and PDAC.

\begin{tabular}{|c|c|c|c|c|c|c|}
\hline & Normal pancreatic ducts (\%) & PanIN 1a (\%) & PanIN 1b (\%) & PanIN $1(1 a+1 b)(\%)$ & PanIN 2 (\%) & PanIN 3 (\%) \\
\hline Pancreatitis & $21(19.3 \%)$ & $30(27.5 \%)$ & $30(27.5 \%)$ & $60(55.0 \%)$ & $22(20.2 \%)$ & $6(5.5 \%)$ \\
\hline Pancreatic ductal adenocarcinoma & $14(10.8 \%)$ & $35(26.9 \%)$ & $37(28.5 \%)$ & $72(55.4 \%)$ & $29(22.3 \%)$ & $15(11.5 \%)$ \\
\hline Total number of lesions & $35(14.7 \%)$ & $65(27.2 \%)$ & $67(28.0 \%)$ & $132(55.2 \%)$ & $51(21.3 \%)$ & $21(8.8 \%)$ \\
\hline
\end{tabular}

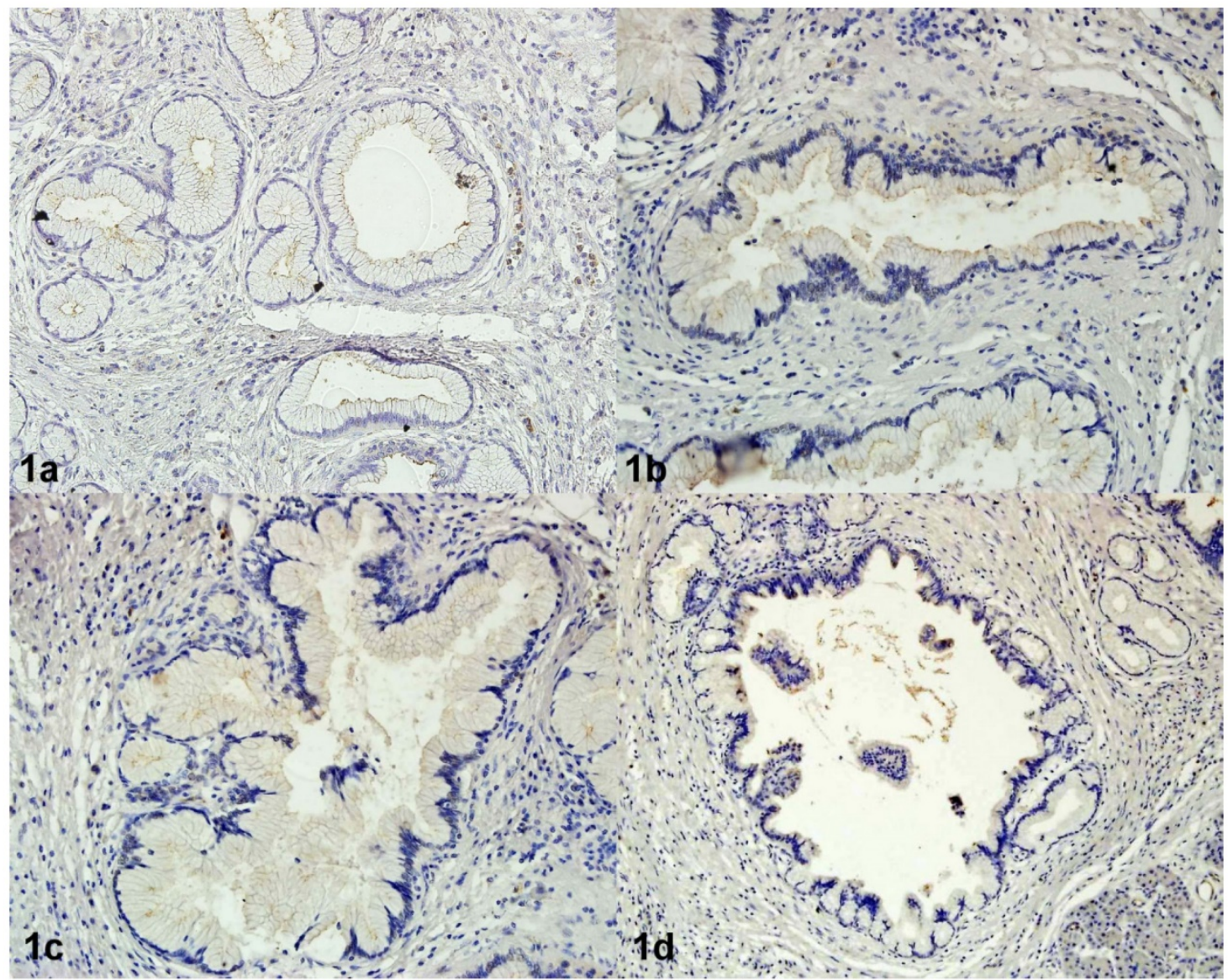

Figure 1. Immunoreactivity of CEACAMI in PanIN IA (1a), in PanIN IB (1b), in PanIN 2 (1c), in PanIN 3 (1d). Magnification 200x 


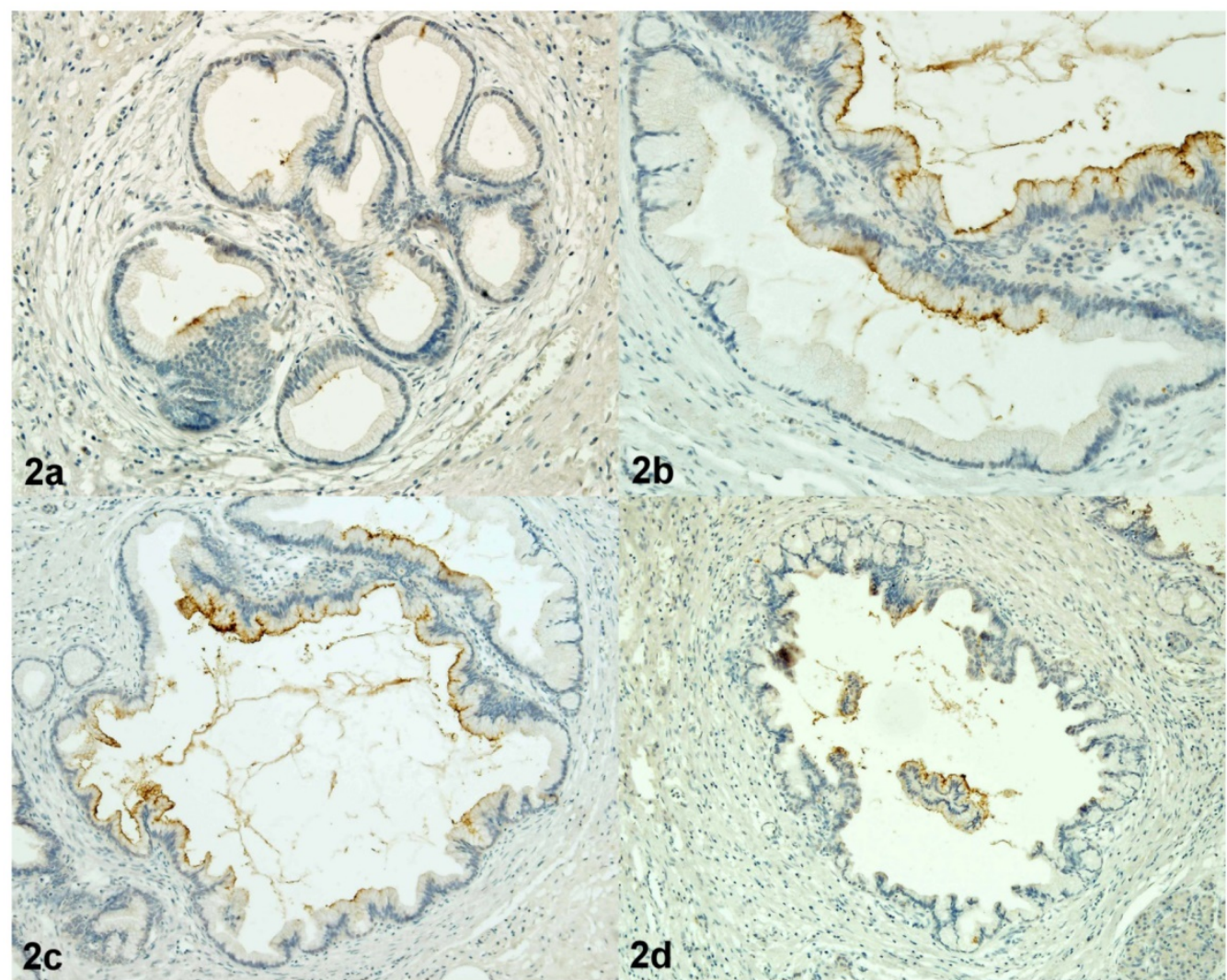

Figure 2. Immunoreactivity of CEACAM5 in PanIN IA (2a), in PanIN IB (2b), in PanIN 2 (2c), in PanIN 3 (2d). Magnification 200x.

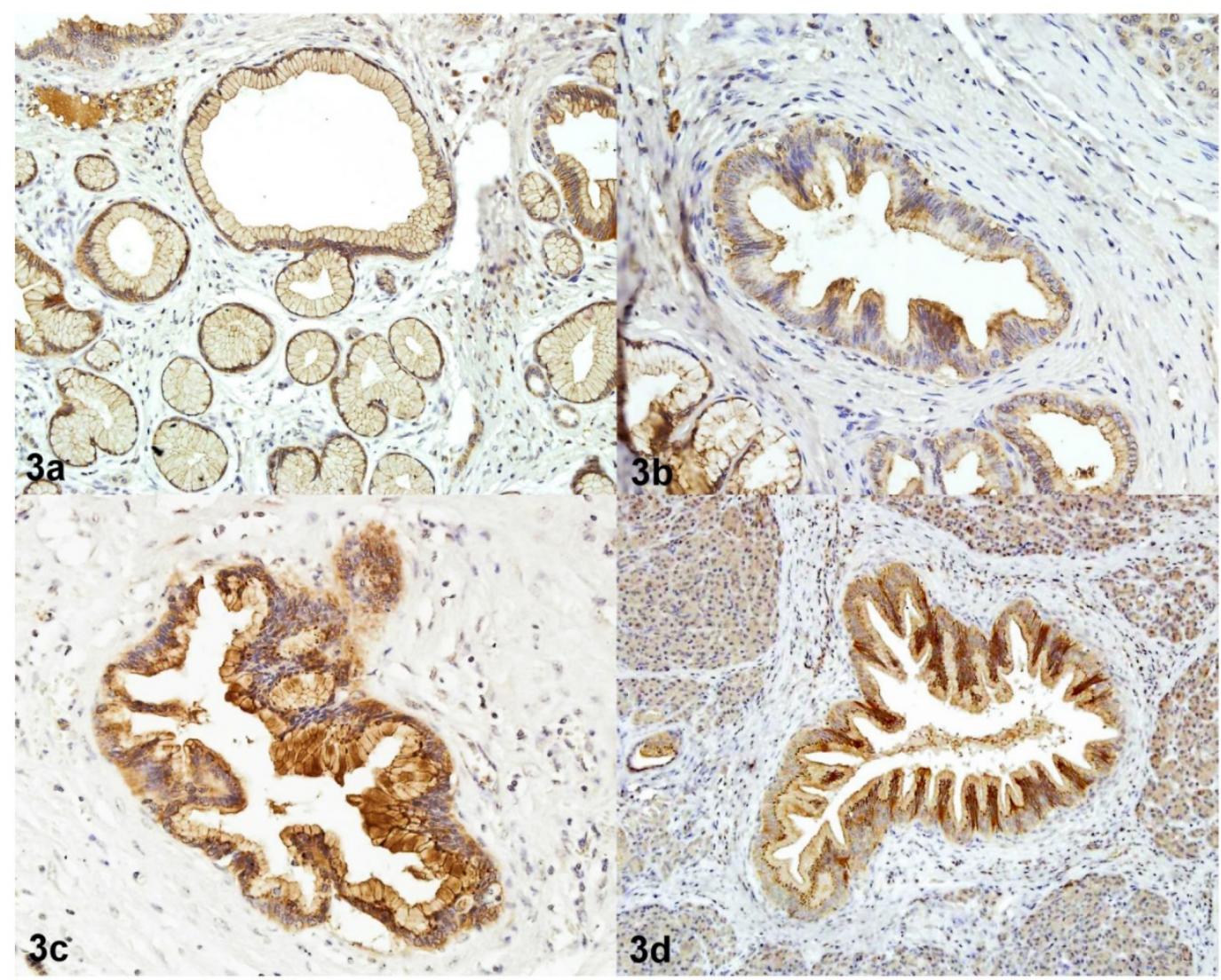

Figure 3. Immunoreactivity of CEACAM6 in PanIN IA (3a), in PanIN 1B (3b), in PanIN 2 (3c), in PanIN 3 (3d) Magnification 200x. 
The associations of CEACAM 1, 5 and 6 expression with clinicopathological parameters has been shown in Table 3. Statistical analysis revealed associations between membrane $(p=0.041)$ and cytoplasm $(p=0.012)$ CEACAM 6 expression with gender. Membrane and cytoplasmic CEACAM 6 expression was higher among females than in males. The percentage of PanIN lesions with membrane expression of CEACAM 6 assessed as a strong was $2.5 \%$ in males and $10.5 \%$ in females. Similar results have been received in case of cytoplasmic reaction percentage of PanIN lesions with strong expression was $6.3 \%$ in males and $18.8 \%$ in females. The strong expression of CEACAM 5 was associated with location of PanIN lesions in the pancreas $(p=0.006)$. Most lesions with strong expression localized in the head of pancreas in comparison to other parts. Moreover, expression of CEACAM 5 and CEACAM 6 (both membrane and cytoplasmic) was stronger $(\mathrm{p}=0.012, \quad \mathrm{p}=0.010, \mathrm{p}<0.001)$ in pancreatic intraepithelial neoplasia coexisted with pancreatic ductal adenocarcinoma than in the case of chronic pancreatitis. Pancreatic intraepithelial neoplasia lesions with moderate and strong expression of CEACAM 5 in PDAC constituted $11.3 \%$ and $17.6 \%$ of all founded lesions whereas in pancreatitis reached $4.6 \%$ and $8.4 \%$ of all lesions. In case of pancreatitis, percentage of PanIN lesions without positive staining for CEACAM 5 was higher compared to PanIN in
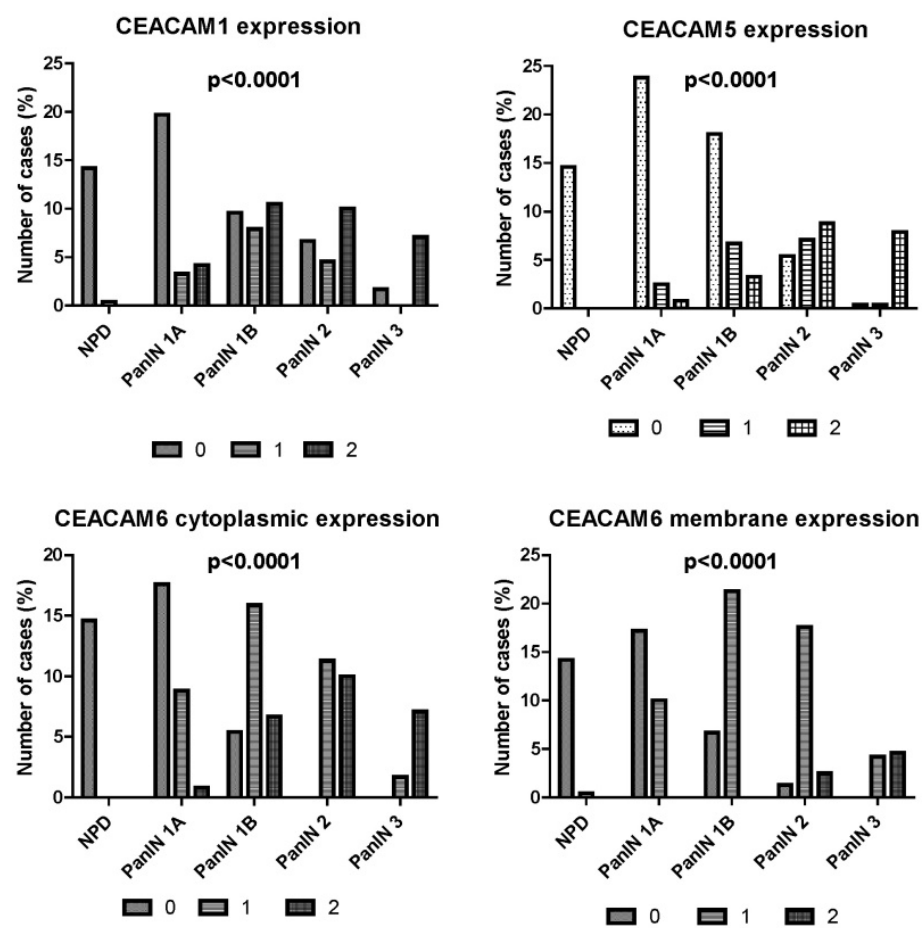

Figure 4. Comparison of the CEACAMI (A), CEACAM5 (B) and CEACAM6 (C, D) expression in various degrees of PanIN.
PDAC $(25.5 \%$ vs. 32.6\%). Similar results were observed for cytoplasmic expression of CEACAM 6. Percentage of lesions with moderate and strong expression were higher in PDAC $(19.7 \%$ and $19.2 \%)$ in comparison with pancreatitis $(17.6 \%$ and $7.2 \%)$. However, a group of PanIN without positive cytoplasmic reaction for CEACAM 6 was more numerous in chronic pancreatitis $(20.1 \%)$ than in PDAC (15.5\%) (Table 3).

\section{Comparison of CEACAM 1, 5 and 6 expression between normal pancreatic ducts and different degrees of pancreatic intraepithelial neoplasia}

The expression of CEACAM1, 5 and 6 was significantly more frequent in pancreatic intraepithelial neoplasia in comparison with normal pancreatic ducts. The expression of these proteins was higher in pancreatic intraepithelial neoplasia compared to the normal tissue $(\mathrm{p}<0.0001)$. Immunohistochemical reaction of CEACAM 1, 5 and 6 was higher in PanIN 1, PanIN 2 and PanIN 3 in comparison with normal pancreatic ducts $(\mathrm{p}<0.0001)$. The expression of CEACAM 1, 5 and 6 occured more frequently in PanIN 2 and PanIN 3 in comparison with PanIN 1 $(\mathrm{p}<0.0001)$. The expression of CEACAM 1, 5 and 6 was also higher in PanIN 3 compared to PanIN 2 lesions (Figure 4).

\section{Correlations between CEACAM 1, 5 and 6 expressions}

The Pearson chi-square test revealed positive correlations between the expressions of CEACAM 1, 5 and $6(\mathrm{p}<0.001)$ in pancreatic intraepithelial neoplasia. This relationship was directly proportional, which means that if the patient had a positive expression of e.g. CEACAM 1 he would also have a positive expression of CEACAM 5 and 6.

\section{Discussion}

Pancreatic cancer is the most lethal cancer because in most cases it is diagnosed at advanced stage, when radical pancreatic resection is impossible. About 95\% of pancreatic cancers constitutes pancreatic ductal adenocarcinoma developed in the exocrine pancreas. It was discovered that PDAC can transform in stepwise progression from precursor lesions referred as pancreatic intraepithelial neoplasia. Pancreatic intraepithelial neoplasia has shown the same spectrum of genetic abnormalities as PDAC, e.g. KRAS mutation, loss CDKN2A function and genetic inactivation of TP53, SMAD4, and BRCA2 [22]. Further investigation of pancreatic intraepithelial neoplasia development may help to detect pancreatic cancer earlier. 
Table 3. Associations of CEACAMI, 5 and 6 expression with chosen clinicopathological parameters.

\begin{tabular}{|c|c|c|c|c|c|c|c|c|c|c|c|c|c|c|c|c|}
\hline \multirow[t]{2}{*}{ Variables } & \multicolumn{4}{|c|}{$\begin{array}{l}\text { CEACAM } 1 \text { expression } \\
(\mathbf{n}, \%)\end{array}$} & \multicolumn{4}{|c|}{$\begin{array}{l}\text { CEACAM } 5 \text { expression } \\
(\mathbf{n}, \%)\end{array}$} & \multicolumn{4}{|c|}{$\begin{array}{c}\text { CEACAM } 6 \text { expression - cytoplasmic } \\
\text { reaction } \\
(\mathrm{n}, \%)\end{array}$} & \multicolumn{4}{|c|}{$\begin{array}{c}\text { CEACAM } 6 \text { expression - membrane } \\
\text { reaction } \\
(\mathbf{n}, \%)\end{array}$} \\
\hline & 0 & 1 & 2 & $p$ & 0 & 1 & 2 & $p$ & 0 & 1 & 2 & $p$ & 0 & 1 & 2 & $p$ \\
\hline $\begin{array}{l}\text { Age (years) } \\
<60 \\
>60\end{array}$ & $\begin{array}{c}53 \\
(22.2) \\
70 \\
(29.2)\end{array}$ & $\begin{array}{c}15 \\
(6.3) \\
21 \\
(8.8)\end{array}$ & $\begin{array}{c}27 \\
(11.3) \\
53 \\
(22.2)\end{array}$ & 0.549 & $\begin{array}{c}62 \\
(25.9) \\
77 \\
(32.2)\end{array}$ & $\begin{array}{c}15 \\
(6.3) \\
28 \\
(11.7)\end{array}$ & $\begin{array}{c}18 \\
(7.5) \\
39 \\
(16.4)\end{array}$ & 0.211 & $\begin{array}{c}43 \\
(18.0) \\
45 \\
(18.8)\end{array}$ & $\begin{array}{c}36 \\
(15.1) \\
56 \\
(23.4)\end{array}$ & $\begin{array}{c}16 \\
(6.7) \\
43 \\
(18.0)\end{array}$ & 0.055 & $\begin{array}{c}39 \\
(16.3) \\
55 \\
(23.0)\end{array}$ & $\begin{array}{c}51 \\
(21.3) \\
75 \\
(31.4)\end{array}$ & $\begin{array}{c}5 \\
(2.1) \\
14 \\
(5.9)\end{array}$ & 0.485 \\
\hline $\begin{array}{l}\text { Gender } \\
\text { male } \\
\text { female }\end{array}$ & $\begin{array}{c}49 \\
(20.5) \\
75 \\
(31.5)\end{array}$ & $\begin{array}{c}16 \\
(6.7) \\
24 \\
(10.0)\end{array}$ & $\begin{array}{c}40 \\
(16.7) \\
35 \\
(14.6)\end{array}$ & 0.296 & $\begin{array}{c}69 \\
(28.9) \\
71 \\
(29.7)\end{array}$ & $\begin{array}{c}15 \\
(6.3) \\
28 \\
(11.7)\end{array}$ & $\begin{array}{c}21 \\
(8.8) \\
35 \\
(14.6)\end{array}$ & 0.227 & $\begin{array}{c}46 \\
(19.2) \\
41 \\
(17.2)\end{array}$ & $\begin{array}{c}44 \\
(18.4) \\
48 \\
(20.1)\end{array}$ & $\begin{array}{c}15 \\
(6.3) \\
45 \\
(18.8)\end{array}$ & 0.012 & $\begin{array}{c}44 \\
(18.4) \\
52 \\
(21.8)\end{array}$ & $\begin{array}{c}55 \\
(23.0) \\
57 \\
(23.8)\end{array}$ & $\begin{array}{c}6 \\
(2.5) \\
25 \\
(10.5)\end{array}$ & 0.041 \\
\hline $\begin{array}{l}\begin{array}{l}\text { Location } \\
\text { head }\end{array} \\
\text { corpus } \\
\text { tail } \\
\text { tail and } \\
\text { corpus }\end{array}$ & $\begin{array}{c}68 \\
(28.5) \\
13 \\
(5.4) \\
36 \\
(15.1) \\
16 \\
(6.7)\end{array}$ & $\begin{array}{c}12 \\
(5.0) \\
2 \\
(0.8) \\
12 \\
(5.0) \\
12 \\
(5.0)\end{array}$ & $\begin{array}{c}32 \\
(13.4) \\
6 \\
(2.5) \\
10 \\
(4.2) \\
20 \\
(8.4)\end{array}$ & 0.057 & $\begin{array}{c}61 \\
(25.5) \\
5 \\
(2.1) \\
38 \\
(15.9) \\
29 \\
(12.1)\end{array}$ & $\begin{array}{c}11 \\
(4.6) \\
7 \\
(2.9) \\
10 \\
(4.2) \\
12 \\
(5.0)\end{array}$ & $\begin{array}{c}40 \\
(16.7) \\
9 \\
(3.8) \\
10 \\
(4.2) \\
7 \\
(2.9)\end{array}$ & 0.006 & $\begin{array}{c}38 \\
(15.9) \\
5 \\
(2.1) \\
19 \\
(7.9) \\
22 \\
(9.2)\end{array}$ & $\begin{array}{c}43 \\
(18.0) \\
8 \\
(3.3) \\
19 \\
(7.9) \\
18 \\
(7.5)\end{array}$ & $\begin{array}{c}31 \\
(13.0) \\
8 \\
(3.3) \\
20 \\
(8.4) \\
8 \\
(3.3)\end{array}$ & 0.483 & $\begin{array}{c}47 \\
(19.7) \\
7 \\
(2.9) \\
21 \\
(8.8) \\
14 \\
(5.9)\end{array}$ & $\begin{array}{c}55 \\
(23.0) \\
8 \\
(3.3) \\
32 \\
(13.4) \\
26 \\
(10.9)\end{array}$ & $\begin{array}{c}10 \\
(4.2) \\
6 \\
(2.5) \\
5 \\
(2.1) \\
8 \\
(3.3)\end{array}$ & 0.454 \\
\hline $\begin{array}{l}\text { Diagnosis } \\
\text { PDAC } \\
\text { chronic } \\
\text { pancreatitis }\end{array}$ & $\begin{array}{c}71 \\
(29.7) \\
61 \\
(25.5)\end{array}$ & $\begin{array}{c}20 \\
(8.4) \\
16 \\
(6.7)\end{array}$ & $\begin{array}{c}39 \\
(16.3) \\
32 \\
(13.4)\end{array}$ & 0.901 & $\begin{array}{c}61 \\
(25.5) \\
78 \\
(32.6)\end{array}$ & $\begin{array}{c}27 \\
(11.3) \\
11 \\
(4 .\end{array}$ & $\begin{array}{c}42 \\
(17.6) \\
20 \\
(8.4)\end{array}$ & 0.012 & $\begin{array}{c}37 \\
(15.5) \\
48 \\
(20.1)\end{array}$ & $\begin{array}{c}47 \\
(19.7) \\
42 \\
(17.6)\end{array}$ & $\begin{array}{c}46 \\
(19.2) \\
19 \\
(7.9)\end{array}$ & 0.010 & $\begin{array}{c}50 \\
(20.9) \\
38 \\
(15.9)\end{array}$ & $\begin{array}{c}76 \\
(31.8) \\
49 \\
(20.5)\end{array}$ & $\begin{array}{c}4 \\
(1.7) \\
22 \\
(9.2)\end{array}$ & $<0.001$ \\
\hline $\begin{array}{l}\text { PanIN } \\
\text { normal } \\
\text { ducts } \\
1 \mathrm{~A} \\
1 \mathrm{~B} \\
2\end{array}$ & $\begin{array}{c}34 \\
(14.2) \\
47 \\
(19.7) \\
23 \\
(9.6) \\
16 \\
(6.7) \\
4 \\
(1.7)\end{array}$ & $\begin{array}{c}1 \\
(0.4) \\
8 \\
(3.3) \\
19 \\
(7.9) \\
11 \\
(4.6) \\
-\end{array}$ & $\begin{array}{c}- \\
\\
10 \\
(4.2) \\
25 \\
(10.5) \\
24 \\
(10.0) \\
17 \\
(7.1)\end{array}$ & $<0.0001$ & $\begin{array}{c}35 \\
(14.6) \\
57 \\
(23.8) \\
43 \\
(18.0) \\
13 \\
(5.4) \\
1 \\
(0.4)\end{array}$ & $\begin{array}{c}- \\
\\
6 \\
(2.5) \\
16 \\
(6.7) \\
17 \\
(7.1) \\
1 \\
(0.4)\end{array}$ & $\begin{array}{c}- \\
\\
2 \\
(0.8) \\
8 \\
(3.3) \\
21 \\
(8.8) \\
19 \\
(7.9)\end{array}$ & $<0.0001$ & $\begin{array}{c}35 \\
(14.6) \\
42 \\
(17.6) \\
13 \\
(5.4) \\
-\end{array}$ & $\begin{array}{c}- \\
\\
21 \\
(8.8) \\
38 \\
(15.9) \\
27 \\
(11.3) \\
4 \\
(1.7)\end{array}$ & $\begin{array}{c}- \\
\\
2 \\
(0.8) \\
16 \\
(6.7) \\
24 \\
(10.0) \\
17 \\
(7.1)\end{array}$ & $<0.0001$ & $\begin{array}{c}34 \\
(14.2) \\
41 \\
(17.2) \\
16 \\
(6.7) \\
3 \\
(1.3) \\
-\end{array}$ & $\begin{array}{c}1 \\
(0.4) \\
24 \\
(10.0) \\
51 \\
(21.3) \\
42 \\
(17.6) \\
10 \\
(4.2)\end{array}$ & $\begin{array}{c}- \\
- \\
- \\
- \\
6 \\
(2.5) \\
11 \\
(4.6)\end{array}$ & $<0.0001$ \\
\hline
\end{tabular}

CEACAM are cell adhesion molecules that participate in generating and maintaining normal tissue architecture and cellular polarity, but they also play an important role in invasion and progression of cancer. Malignant tumors and precancerous lesions are characterized by disruption of tissue architecture and abnormal differentiation. The process of cell differentiation is associated with the loss of cell-cell binding and alterations in the expression of cell adhesion molecules. These are also features of the invasive potential of malignant tumors. Jinka et al. [23] indicated that changes in cell-cell and cell-matrix interactions may be responsible for the ability of cancer cells to cross tissue boundaries and to spread to distant sites [23]. In our work we assessed the expression of CEACAM1, 5 and 6 in pancreatic intraepithelial neoplasia in a group of patients with chronic pancreatitis and pancreatic ductal adenocarcinoma.

One of the molecules responsible for the maintenance of normal tissue architecture is CEACAM1 which $\mathrm{N}$-terminal immunoglobulin domain mediates cell-cell adhesion. CEACAM1 may acts on the cell surface as a sensor molecule to regulate cellular signaling. Moreover, CEACAM1 is a substrate of the insulin receptor and its activation by tyrosine kinase of the insulin receptor after insulin binding begins a cascade of signaling pathways to induce cell growth and proliferation. In addition to the control of cellular proliferation, CEACAM1 may regulate apoptosis, promotes angiogenesis and cellular metastasis, mediates bacterial adhesion and internalization as a pathogen receptor, and even adjusts immune response [24]. So far, function of CEACAM1 in precancerous lesions of pancreas has not been established, although there are several studies assessing immunohistochemical and serum expression of chosen CEACAM molecules in course of different cancers e.g. in colon, breast and lung cancer $[8,9,11,18,25]$. In our immunohistochemical analysis we found a positive expression of CEACAM1 in majority of pancreatic intraepithelial neoplasia lesions. Expression of CEACAM1 was positive both in the group of patients with chronic pancreatitis and 
pancreatic ductal adenocarcinoma without any statistically significant differences between both groups. We observed statistically significant increase in CEACAM1 expression in different degrees of pancreatic intraepithelial neoplasia. It may be associated with transformation of epithelial cells from normal cuboidal cells of epithelium to higher degree of pancreatic intraepithelial neoplasia - PanIN 3. Similar results have been showed by Simeone et al. [25]. They observed positive expression of CEACAM1 in 9\% cases of PanIN 1A, 22\% cases of PanIN 2 and $85 \%$ cases of PanIN 3. Moreover, Simeone et al. [25] evaluated CEACAM1 levels in sera patients with pancreatic ductal adenocarcinoma and compared obtained results with group of healthy people. They showed significantly higher level of CEACAM1 in patients with pancreatic ductal adenocarcinoma and concluded that CEACAM1 may be a novel biomarker for pancreatic cancer. Additionally, their results of positive CEACAM1 expression in precancerous lesions - in pancreatic intraepithelial neoplasia - may support the possibility that CEACAM1 may be used in identifying patients with early stage disease [25]. On the other hand, Simonetti et al. [26] examined the expression of CEACAM1 in normal tissue, dysplastic lesions, oral squamous cell carcinoma and carcinoma in situ. They observed positive expression in normal tissue, while it was negative in dysplastic lesions. These authors suggest that CEACAM1 expression changes dynamically and is reduced in early stages of many cancers [26]. Some authors described decreased expression of CEACAM1 in colon, endometrium, breast and prostate cancers and suggested that this molecule may act as a tumor suppressor and maintains the normal phenotype of epithelial cells [7]. On the other hand, other authors observed the overexpression of CEACAM1 in melanoma, oral and lung cancers and showed an association between the expression of this protein with poor prognosis. Moreover, they concluded that overexpression of CEACAM1 in these cancers may be a marker for progression and metastasis [10, 27, 28]. These observations support a hypothesis that expression of CEACAM1 depends on the type of cancer and is associated with prognosis [29].

CEACAM5, similarly as CEACAM1, takes part in homophilic and heterophilic (CEA binding to non-CEA molecules) interactions indicating a role of this molecule in intercellular adhesion which is involved in cancer invasion and metastasis [15]. Moreover, CEACAM5 acts as a chemoattractant and may promote the metastatic potential in some experimental tumors. Supression of CEACAM5 expression decreases metastases formation in mouse experimental models, whereas anti-CEACAM5 intact
mAb, MN-14, also delayed cellular death due to metastasis in a human colorectal cancer model [14]. Overexpression of CEACAM 5 occurs in about $90 \%$ of gastrointestinal, colorectal and pancreatic cancers, $70 \%$ of non-small cell lung cancers and $50 \%$ of breast cancers [19]. Expression of CEACAM5 has been extensively studied in various cancers and in gastric non-neoplastic and neoplastic lesions but no in the precancerous lesions of the pancreas. In our work we assessed the expression of CEACAM5 in pancreatic intraepithelial neoplasia and we observed that the expression of this protein increased with increasing degree of PanIN and was absent in normal pancreatic ducts. CEACAM5 expression was significantly higher in PanIN coexisted with pancreatic ductal adenocarcinoma than in pancreatitis. Additionally, we showed that CEACAM5 expression was associated with presence of CEACAM6 expression. Similar results have been observed by Gebauer et al. [8] but they evaluated expression of CEACAM1, 5 and 6 in pancreatic ductal adenocarcinoma. They revealed an association between CEACAM5 and 6 expression as well as between CEACAM5 and positive lymph node status and distant metastasis. Additionally, the Kaplan-Meier survival analysis performed by Gebauer et al. [8] showed that elevated CEACAM5 and/or 6 expression in patients with PDAC was connected with shortened overall survival and disease free survival. As regards to Gebauer's [8] and our results we may conclude that overexpression of CEACAM5 present in different degrees of pancreatic intraepithelial neoplasia may be an unfavorable prognostic factor [8]. Blumenthal et al. [15] evaluated CEACAM5 expression in colon, ovary, breast, lung, prostate and pancreatic cancers. In their work, independently from type of tumor cell differentiation, CEACAM5 expression was higher in pancreatic ductal adenocarcinoma than in non-neoplastic pancreas. Similarly, in our study, CEACAM5 expression was absent in normal pancreatic ducts and increased with increasing degree of PanIN which is precursor lesion of pancreatic ductal adenocarcinoma. We also assessed the expression of CEACAM6 in pancreatic intraepithelial neoplasia and we observed simultaneously cytoplasmic and membrane reaction in PanIN. A statistically significant increase in the cytoplasmic and membrane expression of CEACAM6 following the increasing stage of pancreatic intraepithelial neoplasia was showed in our study. Additionally, cytoplasmic expression was stronger than membrane. We also observed a statistically significant relationship between cytoplasmic and membrane level of CEACAM6 with disease, since the expression of CEACAM6 was stronger in patients with pancreatic ductal adenocarcinoma than in 
pancreatitis. Many authors confirmed that CEACAM6 expression is positive not only in cancer but also in inflammatory tissue. It may be caused by pro-inflammatory properties of CEACAM [8]. Moreover, CEACAM6 may inhibit cell differentiation and cell polarization, and it also causes disruption of tissue architecture. Overexpression of CEACAM6 modulates tumor progression in terms of cell invasion and metastasis, and represents an acquired advantage of tumor cells directly responsible for an invasive phenotype [30]. Overexpression of CEACAM5 and CEACAM6 in pancreatic ductal adenocarcinoma is associated with greater resistance to anoikis and increased metastatic ability. However, posttranscriptional inhibition of CEACAM5 and CEACAM6 expression reduce the potential of pancreatic adenocarcinoma cells to resist anoikis and liver metastasizing. Resistance to anoikis is a property of transformed cells and a determinant of tumorigenesis and metastasis [31]. Duxbury et al. [32] also assessed CEACAM6 expression in pancreatic intraepithelial neoplasia and observed that $66 \%$ of PanIN 1A had no CEACAM6 expression. In their results $65 \%$ of PanIN 1B demonstrated weak CEACAM6 staining. Simultaneously, the prevalence of CEACAM6 expression was similar among PanIN 2 and PanIN 1B lesions, however, the staining was more intense among PanIN 2. All of the PanIN 3 lesions expressed CEACAM6 at moderate to high levels. Similarly as in our paper, increased expression of CEACAM6 following the increasing degree of pancreatic intraepithelial neoplasia was statistically significant. Duxbury et al. [18] concluded that CEACAM6 is not only a potentially useful biomarker, but may participate in oncogenic changes that occur during progression from PanIN lesions to invasive pancreatic cancer. In other study regarding assessment of CEACAM6 expression in pancreatic cancer, Duxbury et al. [18, 31, 32] showed that overexpression of CEACAM6 increases cellular invasiveness but posttranscriptional silencing of CEACAM6 expression decreases the invasiveness of pancreatic adenocarcinoma cells. In our paper CEACAM6 expression increases with increasing of stage of pancreatic intraepithelial neoplasia. Thus we suppose that this molecule is associated with transformation of pancreatic ductal epithelial cells in pancreatic intraepithelial neoplasia and may promote a transformation of benign lesions into an aggressive invasive carcinoma.

In summary, positive expression of CEACAM1, 5 and 6 appears to be an early event in progression from normal pancreatic ductal epithelium to pancreatic intraepithelial neoplasia. It is supposed that CEACAM molecules may play an important role in the transformation of epithelial cells. Furthermore, these molecules may become in future a potential marker that will help in the identification of precancerous lesions in the pancreas.

\section{Competing Interests}

The authors have declared that no competing interest exists.

\section{References}

1. Society AC. Cancer facts \& figures 2017. Atlanta, American Cancer Society, 2017.

2. Garrido-Laguna I, Hidalgo M. Pancreatic cancer: From state-of-the-art treatments to promising novel therapies. Nat Rev Clin Oncol 2015;12:319-34.

3. Hidalgo M, Cascinu S, Kleeff J, et al. Addressing the challenges of pancreatic cancer: Future directions for improving outcomes. Pancreatology 2015;15:8-18

4. Haugk B. Pancreatic intraepithelial neoplasia-can we detect early pancreatic cancer? Histopathology 2010;57:503-14.

5. Hruban RH, Adsay NV, Albores-Saavedra J, et al. Pancreatic intraepithelial neoplasia: A new nomenclature and classification system for pancreatic duct lesions. Am J Surg Pathol 2001;25:579-86.

6. Kuespert K, Pils S, Hauck CR. Ceacams: Their role in physiology and pathophysiology. Curr Opin Cell Biol 2006;18:565-71.

7. Beauchemin N, Arabzadeh A. Carcinoembryonic antigen-related cell adhesion molecules (ceacams) in cancer progression and metastasis. Cancer Metastasis Rev 2013;32:643-71.

8. Gebauer F, Wicklein D, Horst J, et al. Carcinoembryonic antigen-related cell adhesion molecules (ceacam) 1, 5 and 6 as biomarkers in pancreatic cancer. PLoS One 2014;9:e113023.

9. Thöm I, Schult-Kronefeld O, Burkholder I, et al. Expression of ceacam-1 in pulmonary adenocarcinomas and their metastases. Anticancer Res 2009;29:249-54.

10. Thies A, Moll I, Berger J, et al. Ceacam1 expression in cutaneous malignant melanoma predicts the development of metastatic disease. J Clin Oncol 2002;20:2530-6.

11. Dango S, Sienel W, Schreiber $M$, et al. Elevated expression of carcinoembryonic antigen-related cell adhesion molecule 1 (ceacam-1) is associated with increased angiogenic potential in non-small-cell lung cancer. Lung Cancer 2008;60:426-33.

12. Prall F, Nollau P, Neumaier M, et al. Cd66a (bgp), an adhesion molecule of the carcinoembryonic antigen family, is expressed in epithelium, endothelium, and myeloid cells in a wide range of normal human tissues. J Histochem Cytochem 1996;44:35-41.

13. Hanahan D, Weinberg RA. Hallmarks of cancer: The next generation. Cell 2011;144:646-74.

14. Blumenthal RD, Hansen $\mathrm{HJ}$, Goldenberg DM. Inhibition of adhesion, invasion, and metastasis by antibodies targeting ceacam6 (nca-90) and ceacam5 (carcinoembryonic antigen). Cancer Res 2005;65:8809-17.

15. Blumenthal RD, Leon E, Hansen HJ, et al. Expression patterns of ceacam5 and ceacam6 in primary and metastatic cancers. BMC Cancer 2007;7:2.

16. Ordoñez C, Screaton RA, Ilantzis C, et al. Human carcinoembryonic antigen functions as a general inhibitor of anoikis. Cancer Res 2000;60:3419-24.

17. Shanmugathasan M, Jothy S. Apoptosis, anoikis and their relevance to the pathobiology of colon cancer. Pathol Int 2000;50:273-9.

18. Duxbury MS, Matros E, Clancy T, et al. Ceacam6 is a novel biomarker in pancreatic adenocarcinoma and panin lesions. Ann Surg 2005;241:491-6.

19. Zhou J, Fan X, Chen N, et al. Identification of ceacam5 as a biomarker for prewarning and prognosis in gastric cancer. J Histochem Cytochem 2015;63:922-30.

20. Kim KS, Kim JT, Lee SJ, et al. Overexpression and clinical significance of carcinoembryonic antigen-related cell adhesion molecule 6 in colorectal cancer. Clin Chim Acta 2013;415:12-9.

21. Kern S, Hruban R, Hollingsworth MA, et al. A white paper: The product of a pancreas cancer think tank. Cancer Res 2001;61:4923-32.

22. He XY, Yuan YZ: Advances in pancreatic cancer research: Moving towards early detection. World J Gastroenterol 2014;20:11241-8.

23. Jinka R, Kapoor R, Sistla PG, et al. Alterations in cell-extracellular matrix interactions during progression of cancers. Int J Cell Biol 2012;2012:219196.

24. Ling Y, Wang J, Wang L, et al. Roles of ceacam1 in cell communication and signaling of lung cancer and other diseases. Cancer Metastasis Rev 2015;34:347-57

25. Simeone DM, Ji B, Banerjee M, et al. Ceacam1, a novel serum biomarker for pancreatic cancer. Pancreas 2007;34:436-43.

26. Simonetti O, Lucarini G, Rubini C, et al. Correlation between immunohistochemical staining of ceacam1 and clinicopathological findings in oral pre-neoplastic lesions and squamous cell carcinoma. Med Mol Morphol 2018;51:41-7. 
27. Zhou CJ, Qu X, Yang YM, et al. Ceacam1 distribution and it's effects on angiogenesis and lymphangiogenesis in oral carcinoma. Oral Oncol 2009; 45:883-6.

28. Laack E, Nikbakht H, Peters A, et al. Expression of ceacam1 in adenocarcinoma of the lung: A factor of independent prognostic significance. J Clin Oncol 2002;20:4279-84.

29. Fiori V, Magnani M, Cianfriglia M. The expression and modulation of ceacam1 and tumor cell transformation. Ann Ist Super Sanita 2012;48:161-71.

30. Rizeq B, Zakaria Z, Ouhtit A. Towards understanding the mechanisms of actions of carcinoembryonic antigen-related cell adhesion molecule 6 in cancer progression. Cancer Sci 2018;109:33-42.

31. Duxbury MS, Ito H, Benoit E, et al. Ceacam6 is a determinant of pancreatic adenocarcinoma cellular invasiveness. Br J Cancer 2004;91:1384-90.

32. Duxbury MS, Ito H, Zinner MJ, et al. Ceacam6 gene silencing impairs anoikis resistance and in vivo metastatic ability of pancreatic adenocarcinoma cells. Oncogene 2004;23:465-73 